\title{
DESEMPEÑO DE Sitophilus zeamais (COLEOPTERA: CURCULIONIDAE) EN DIFERENTES VARIEDADES DE MAÍZ Y CONDICIONES ATMOSFÉRICAS
}

\author{
Adalberto Hipólito de Sousa \\ Mestrando em Entomologia da Universidade Federal de Viçosa - Departamento de Biologia Animal, 36570-000, \\ Viçosa, MG, Brasil. adalberto@insecta.ufv.br. \\ Patrício Borges Maracajá \\ Prof. Dr. Departamento de Ciências Vegetais da UFERSA - 59600-970, Mossoró-RN, patrício@ufersa.edubr
}

Alexandre Almeida da Costa

Estudante de Agronomia - Departamento de Ciências Vegetais da UFERSA- 59600-970, Mossoró, RN-Brasil.

alexandreac1@hotmail.com

Alberto Soto Giraldo

Prof. Departamento de Fitotecnia - Universidade de Caldas - Colômbia. Doutorando em Entomologia - UFV.

Tadeu Fladiner Costa Pereira

Departamento de Ciência Vegetais - UFERSA - Universidade Federal Rural do Semi-Árido e-mail:

fladner@gmail.com

\begin{abstract}
RESUMEN - El objetivo de este trabajo fue investigar el potencial de desarrollo y de destrucción del gorgojo Sitophilus zeamais Mots. (Coleoptera: Curculionidae) en tres vari-edades de maíz (cruzeta, milhometro e hibra) almacenadas en tres condiciones atmosféricas (hermética, semihermética y no-hermética). Fueron evaluadas al final del período de almacenamiento, 60 días, a emergencia de adultos y a perdida de peso de los granos. La variedad milhometro se mostró más susceptible al desarrollo del gorgojo, pues presentó los mayores valores de emergencia de adultos y perdida de peso de los granos en las condiciones semi-hermética y no-hermética. Ya la variedad cruzeta, al ser almacenada en la condición hermética, presentó menor emergencia de adultos y perdida de peso de granos, tornando, por tanto, evidente la influencia de la condición hermética en la biología de esta especie, pues proporcionó menor incidencia de insectos y pérdida de peso del producto lo mismo en las variedades que se mostraron más susceptibles.
\end{abstract}

Palabras clave: Gorgojo, resistencia de plantas, almacenamiento.

\section{ACTING OF Sitophilus zeamais (COLEOPTERA: CURCULIONIDAE) IN DIFFERENT VARIETIES OF CORN AND ATMOSPHERIC CONDITIONS}

SUMMARY - The objective of this work was to investigate the development potential and of destruction of the weevil Sitophilus zeamais Mots. (Coleoptera: Curculionidae) in three corn varieties (crosspiece, milhometro and hibra) stored in three atmospheric conditions (hermetic, semihermetic and no-hermetic). Were appraised at the end of the storage period, 60 days, the adults' emergency and the loss of weight of the grains. The variety milhometro was shown more favorable to the development of the weevil, because it presented the largest values of adults' emergency and loss of weight of the grains in the semi-hermetic and no-hermetic conditions. Already the variety crosspiece, when being stored in the hermetic condition, presented smaller emergency of adults and loss of weight of grains, turning, therefore, evident it influences her/it of the hermetic condition in 
the biology of this species, because it provided smaller incidence of insects and loss of weight of the same product in the varieties that were shown more acceptable.

Key words: Weevil, plant resistance, storage.

\section{INTRODUCCIÓN}

El aumento de la demanda de ali-mentos, en función del crecimiento poblacional, exige el desarrollo y el perfeccionamiento de nuevas técnicas de manejo de granos durante el almacenamiento. Dentro de los factores que interfieren en la calidad de los granos durante el almacenamiento, el ataque severo de plagas se destaca por resultar en mayor pérdida de la calidad del producto final. Entre las plagas asociadas al almacenamiento de maíz, el gorgojo Sitophilus zeamais Mots. (Coleoptera: Curculionidae) se destaca entre las mas importantes por atacar granos intactos. Esta especie es semejante al S. oryzae (Lineu 1763), pudiendo ser diferenciados apenas por la genitalia (Rees 1996; Lorini 2002). En $S$. zeamais la superficie exterior del pene tiene una elevación central entre dos depresiones longitudinales ligeramente aplanadas, en cuanto en $S$. oryzae es completamente liso y convexo (Rees 1996).

El control de estos insectos ha sido realizado en larga escala con el uso de productos químicos, donde el expurgo con fosfato de alumínio, cuyo principio activo es la fosfina, es la técnica mas empleada en el control de estos insectos, y que representa un grande riesgo para el almacenamiento, ya que son muchos los relatos que muestran el desarrollo de resistencia de éstos insectos a la fosfina. Dentro de las diferentes formas de minimizar el ataque de las plagas y de disminuir la presión de selección de individuos resistentes a fosfina, la utilización de materiales resistentes presenta una serie de ventajas, pues posee la resistencia contra insectos incorporada a su genoma, proporciona menores costos y no afecta el equilibrio ambiental (Lara 1991; Guzzo et al. 2002). Variedades que expresan resistencia tienen atributos físicos o bioquímicos que modifican las respuestas de comportamiento o que afectan el desarrollo o la sobrevivencia de las especies de insecto por aberraciones metabólicas (Throne et al. 2000). El maíz por presentar elevada variabilidad genética, se torna un óptimo objeto de estudio de selección y mejoramiento de genotipos resistentes a insectos plaga durante el almacenamiento, donde puede haber asociación con otras técnicas de control de insectos, como la modificación atmosférica del ambiente en que se encuentra la masa de granos.

El almacenamiento hermético de granos es una forma de atmósfera modificada, en que el grano, por si solo, a través de la respiración, crea una atmósfera rica en dióxido de carbono y baja en oxigeno. Esta técnica pudo haber sido usada por los egipcios sécalos atrás y persiste hasta hoy en África, donde el almacenamiento hermético es ampliamente utilizado en el almacenamiento de granos (White y Leesh 1996). El resultado de esta estrategia de control se debe al hecho de alcanzar directamente el sistema respiratorio del insecto en su demanda por oxigeno y, al mismo tiempo, aprovechar el efecto nocivo de la alta concentración de $\mathrm{CO}_{2}$ (Faroni et al. 2002).

Por lo tanto, considerando los problemas que representa la incidencia de $S$. zeamais para el almacenamiento de maíz, el objetivo de este trabajo fue investigar el potencial de desarrollo y de destrucción de este insecto en tres variedades de maíz almacenadas en tres condiciones atmosféricas.

\section{MATERIALES Y MÉTODOS}

Los experimentos fueron conduci-dos en el Laboratorio de Control de Insectos del Departamento de Ciencias Vegeta-les de la Universidad Federal Rural do Semi-Árido, UFERSA/RN/Brasil, en cámaras climáticas del 
tipo BOD, a tempera-tura de $27 \pm 2{ }^{\circ} \mathrm{C}$ y humedad relativa $65 \pm 5 \%$. Fueron probadas tres variedades de maíz (cruzeta, milhometro e hibra) comercializadas en el estado de Rio Grande do Norte, Brasil. Estas variedades fueron almacenadas en las condiciones atmosféricas herméticas, semi-hemética y no hermética. Para obtención de estas condiciones el maíz fue almacenado en garrafas plásticas lacradas (condición hermética), potes de hierro tapados (condición semi-hermética) y sacos de tejido de algodón (condición no-hermética).

Después del acondicionamiento de los granos en los embalajes, se hizo la infestación de los granos con 20 ejemplares adultos de $S$. zeamais provenientes de la cría masal del propio laboratorio, donde permanecieron durante todo el período de almacenamiento, 60 días, tiempo necesario para que la progenie de los insectos completaran su ciclo y actuaran sobre la masa de granos. El hecho que la colecta de datos se realizó en este período de almacenamiento, apenas a la generación $\mathrm{F}_{1}$, se debió a la decisión de analizar valores mayores sobre el desarrollo de los insectos en las diferentes variedades investigadas en cada condición atmosférica.

La unidad experimental fue compuesta de 50 granos y 20 insectos para cada variedad y condición atmosférica. Fue utilizado un diseño experimental completamente al azar, con 4 repeticiones en es-quema factorial $3 \times 3$, donde el primer factor se refiere a las condiciones atmosféricas, y el segundo factor a las variedades. Fueron evaluados al final del período de almacenamiento el número de insectos adultos de $S$. zeamais emergidos de los granos y la pérdida de peso de los granos ocasionada por la acción de los insectos.

\section{RESULTADOS Y DISCUSIÓN}

Interacción significativa entre las variedades de maíz y las condiciones atmosféricas fue observada tanto para emergencia de adultos como para la perdida de peso de granos. Desdoblando la interacción variedades dentro de condiciones atmosféricas, se observó que la emergencia de los insectos fue estadísticamente semejante en las tres variedades sobre condiciones herméticas de almacenamiento. Ya en las condiciones semiherméticas y no-herméticas la emergencia de adultos fue significativamente menor en la variedad cruzeta, confiriendo mayor resistencia a esta variedad y mayor eficiencia a condición hermética (Tabla 1).

Con relación a la perdida de peso de granos las variedades cruzeta e hibra tuvieron menores valores en las condiciones semiherméticas y no herméticas, y al contrario del que se observó en la emergencia de adultos, no tuvieron diferencia estadística entre si. Este hecho ciertamente está relacionado al corto período de almacenamiento de los granos, donde se cree que con el prolongamiento del período de almacenamiento la perdida de peso sea significativamente observada, pues los mismos resultados no demostraron diferencia estadística; se verifica que en términos absolutos la variedad hibra tuvo mayor pérdida de peso que la variedad cruzeta en la condición nohermética, lo que sugiere que la variedad cruzeta es menos adecuada para el consumo alimentar de los insectos. Ya en la condición hermética las tres variedades no difirieron estadísticamente entre si (Tabla 2), tornando evidente la eficiencia

Tabla 1. Emergencia de adultos de Sitophilus zeamais en tres variedades de maíz después de 60 días de almacenamiento en tres condiciones atmosféricas.

\begin{tabular}{llll}
\hline \multirow{2}{*}{ Variedades } & \multicolumn{3}{l}{ Condiciones atmosféricas } \\
\cline { 2 - 4 } & Hermética & Semi-hermética & No-hermética \\
\hline Cruzeta & $0,50^{1} \mathrm{Aa}$ & $9,00 \mathrm{Ab}$ & $10,25 \mathrm{Ab}$ \\
Hibra & $1,00 \mathrm{Aa}$ & $14,50 \mathrm{Bb}$ & $20,75 \mathrm{Bc}$ \\
Milhometro & $4,50 \mathrm{Aa}$ & $17,75 \mathrm{Bb}$ & $33,50 \mathrm{Cc}$ \\
\hline
\end{tabular}


${ }^{1}$ Medias seguidas por la letra mayúscula en las columnas o por la misma letra minúscula en las líneas no difieren entre si por el test de Tukey ( $\mathrm{P}>$ 0,05). C.V: $23 \cdot 3$

Tabla 2. Pérdida de peso de granos (g) de tres variedades de maíz infestadas con Sitophilus zeamais después de 60 días de almacenamiento en tres tipos de embalajes.

\begin{tabular}{llll}
\hline \multirow{4}{*}{ Variedades } & \multicolumn{3}{l}{ Condiciones atmosféricas } \\
\cline { 2 - 4 } Cruzeta & Hermética & Semi-hermética & No-hermética \\
Hibra & $0,14^{1} \mathrm{Aa}$ & $1,31 \mathrm{Ab}$ & $1,61 \mathrm{Ab}$ \\
Milhometro & $0,20 \mathrm{Aa}$ & $1,31 \mathrm{Ab}$ & $2,12 \mathrm{ABc}$ \\
& $0,00 \mathrm{Aa}$ & $1,94 \mathrm{Bb}$ & $2,22 \mathrm{Bb}$ \\
\hline & \multicolumn{2}{l}{ Medias seguidas por la letra mayúscula en las columnas o por la misma letra } \\
minúscula en las líneas no difieren entre si por el test de Tukey (P $>0,05)$. C.V: \\
27.7
\end{tabular}

de la condición hermética en la biología de esta especie, donde las variedades mas susceptibles al desarrollo del gorgojo presentaron menor incidencia de insectos y menor pérdida de peso de producto.

La menor susceptibilidad de la variedad cruzeta puede estar asociada a antibiosis, pues al principio las tres variedades tuvieron poblaciones de gorgojo del mismo tamaño, y así algún factor ligado a las características del grano pudieron haber afectado el desarrollo de los insectos, resoltando en la reducida población verificada en el final del período de almacenamiento. De esta forma, esta suposición explicaría también la menor perdida de peso de esta variedad, donde el menor número de insectos habría proporcionado menor perdida de peso. La menor susceptibilidad de la variedad Cruzeta pudo aun estar relacionada a la nopreferencia para alimentación u oviposición como forma de resistencia envuelta (Guzzo et al., 2002). Aun con relación a la oviposición, se piensa que diferentes factores químicos pueden estar íntimamente relacionados con el germen de los granos, confiriendo serias implicaciones durante el período de oviposición (Throne et al., 2000). Con relación a las variedades que presentaron patrones de susceptibilidad (hibra y milhometro), hay la posibilidad que liberaran sustancias agradables a los insectos, como olor y sabor (Guzzo et al., 2002).

Boiça et al. (1997); Toscano et al. (1999) y Guzzo et al. (2002), en experimentos con diferentes materiales de maíz, también verificaron diferencias de consumo entre los genotipos investigados, donde asociaron la pérdida total de granos al bajo número de adultos emergidos, donde fue sugerido que algunos genotipos de maíz son menos adecuados al consumo alimentar de los insectos. Con todo, conforme a lo mencionado por Guzzo et al. (2002), es importante saber que cuando el alimento es nutricionalmente adecuado, el insecto puede comer menos y así provocar menor pérdida de granos.

El hecho que los insectos no se desarrollaran bien en las condiciones herméticas ciertamente está relacionado a la atmósfera rica en dióxido de carbono y pobre en oxigeno, pues además de la exclusión de oxigeno para la substitución de aire, el dióxido de carbono también es conocido por ser tóxico en su estado normal y ser aún un poderoso anestésico (Faroni et al. 2002), pudiendo actuar como inhibidor de crecimiento en insectos (Whi-te y Leesh 1996). Los efectos tóxicos del dióxido de carbono son atribuidos al hecho de estimular la abertura de los espiráculos durante la respiración, manteniéndolos abiertos, con eso, aumentando su pérdida de agua (Price 1984, citados por Faroni et al. 2002). Puede aún tener relación con la falta de triglicéridos como substratos para metabolismo de energía (Donahaye 1991; Adler 2000) y una acidificación a nivel celular también podría conducir a un rompimiento de reacciones vitales (Adler 2000). Con todo, la causa directa de la mortalidad de insectos 
expuestos a atmósferas modificadas no está completamente esclarecida.

\section{CONCLUSIONES}

La emergencia de adultos de $S$. zeamais y la pérdida de peso de los granos tuvieron menores valores cuando los granos de la variedad cruzeta fueron almacenados en la condición hermética. Ya la variedad milhometro se mostró más susceptible al desarrollo del gorgojo, pues presen-tó mayores valores de emergencia de adultos y perdida de peso de los granos en las condiciones semi-hermética y nohermética.

\section{AGRADECIMENTOS}

Los autores agradecen a la Universidad Federal Rural do Semi-Árido, en especial al Departamento de Ciencias Vegetales, por el apoyo en la realización de este trabajo.

\section{REFERENCIAS}

ADLER, C.; CORINTH, H. G.; REICH-MUTH, C. 2000. Modified atmospheres. p.104-146. En: SUBRAMANYAM, B.; HAGSTRUM, D. W. Alternatives to pesti-cides in stored-product IMP. Massachu-setts: Kluwer Academic.

BOIÇA, A. L..; LARA, F. M.; GUIDI, F. P. 1997. Resistência de genótipos de milho ao ataque de Sitophilus zeamais (Coleop-tera, Curculionidae). Anais da Sociedade Entomológica do Brasil 26 (3): 481-485.

DONAHAYE, E. 1991. The potential for stored-product insects to develop resis-tance to modified atmospheres. Procee-dings. 5th International Working Confe-rence on StoredProduct. p. 989-998. Fran-ce.
FARONI, L. R. D.; GUEDES, R. N. C.; BERBEERT, P. A.; SILVA, A. P. R. A. 2002. Atmosfera modificada no controle das pragas de grãos armazenados. p. 463-491.En: LORINI, I.; MIIKE, L. H.; SCUSSEL, V. M. Armazenagem de grãos. Volume I. IBG. Campinas. Brasil. $1000 \mathrm{p}$.

GUZZO, E. C.; ALVES, L. F. A.; ZANIN, A.; VENDRAMIN, J. D. 2002. Identifica-ção de materiais de milho resistentes ao ataque de gorgulho Sitophilus zeamais (Mots., 1855) (Coleoptera: Curculionidae). Arquivos do Instituto Biológico 69 (2): 69-73.

LARA, F. M. 1991. Princípios de resistên-cia de plantas a insetos. 2. ed. Agronômica Ceres, 336p.

LORINI, I. 2002. Descrição, biologia e danos das principais pragas de grãos armazenados. p. 381-397. En: LORINI, I.; MIIKE, L. H.; SCUSSEL, V. M. Armaze-nagem de grãos. Volume I. IBG. Campi-nas. Brasil. 1000 p.

PRICE, N. R. 1984. Active exclusion of phosphine as a mechanism of resistance in Rhyzopertha dominica (F.). Journal of Stored Products Research 20 (3): 163-167.

REES, D. P. 1996. Coleoptera. p.1-39. En: SUBRAMANYAM, B.; HAGSTRUM, D. W. Integrated management of insects in stored products. Volume I. Marcel Dek-ker. New York. 426 p.

THRONE, J. E.; BAKER, J. E.; MESSI-NA, F. J.; KRAMER, K. J.; HOWARD, J. A. 2000. Varieal resistance. p.165-192. En: SUBRAMANYAM, B.; HAGSTRUM, D. W. Alternatives to pesticides in stored-product 
IMP. Volume I. Kluwer Acade-mic. ciedade Entomológica Brasileira 28(1): 141Massachusetts. 437 p. 147.

TOSCANO, L. C.; BOIÇA JUNIOR, A. L.; WHITE, N. D. G.; LEESCH, J. G. 1996. LARA, F. M.; WAQUIL, J. M. 1999. Chemical control. p. 287-330. En: SUResistência e mecanismos envolvidos em BRAMANYAM, B.; HAGSTRUM, D. W. genótipos de milho em relação ao ataque do Integrated management of insects in stored gorgulho, Sitophilus Zeamais Mots. products. Volume I. Marcel Dekker. New York. (Coleoptera, Curculionidae). Anais da So- 426 p. 